\title{
Physics of High-Charge Electron Beams in Laser-Plasma Wakefields
}

\author{
J. Götzfried, ${ }^{1,2}$ A. Döpp $\odot,{ }^{1,2,}$ M. F. Gilljohann, ${ }^{1,2}$ F. M. Foerster $\odot,{ }^{1}$ H. Ding $\odot,{ }^{1,2}$ S. Schindler,${ }^{1,2}$ \\ G. Schilling $\oplus^{1}{ }^{1}$ A. Buck, ${ }^{2}$ L. Veisz $\oplus^{2,3}$ and S. Karsch $\oplus^{1,2,{ }^{\prime}}$ \\ ${ }^{1}$ Ludwig-Maximilians-Universität München, Am Coulombwall 1, 85748 Garching, Germany \\ ${ }^{2}$ Max-Planck-Institut für Quantenoptik, Hans-Kopfermann-Strasse 1, 85748 Garching, Germany \\ ${ }^{3}$ Department of Physics, Umeå University, SE-901 87, Umeå, Sweden
}

(Received 27 March 2020; revised 9 June 2020; accepted 1 September 2020; published 21 October 2020)

\begin{abstract}
Laser wakefield acceleration (LWFA) and its particle-driven counterpart, particle or plasma wakefield acceleration (PWFA), are commonly treated as separate, though related, branches of high-gradient plasmabased acceleration. However, novel proposed schemes are increasingly residing at the interface of both concepts where the understanding of their interplay becomes crucial. Here, we present a comprehensive study of this regime, which we may term laser-plasma wakefields. Using datasets of hundreds of shots, we demonstrate the influence of beam loading on the spectral shape of electron bunches. Similar results are obtained using both 100-TW-class and few-cycle lasers, highlighting the scale invariance of the involved physical processes. Furthermore, we probe the interplay of dual electron bunches in the same or in two subsequent plasma periods under the influence of beam loading. We show that, with decreasing laser intensity, beam loading transitions to a beam-dominated regime, where the first bunch acts as the main driver of the wakefield. This transition is evidenced experimentally by a varying acceleration of a lowenergy witness beam with respect to the charge of a high-energy drive beam in a spatially separate gas target. Our results also present an important step in the development of LWFA using controlled injection in a shock front. The electron beams in this study reach record performance in terms of laser-to-beam energy transfer efficiency (up to $10 \%$ ), spectral charge density (regularly exceeding $10 \mathrm{pC} \mathrm{MeV}^{-1}$ ), and angular charge density (beyond $300 \mathrm{pC} \mu \mathrm{sr}^{-1}$ at $220 \mathrm{MeV}$ ). We provide an experimental scaling for the accelerated charge per terawatt (TW) of laser power, which approaches $2 \mathrm{nC}$ at $300 \mathrm{TW}$. With the expanding availability of petawatt-class (PW) lasers, these beam parameters will become widely accessible. Thus, the physics of laser-plasma wakefields is expected to become increasingly relevant, as it provides new paths toward low-emittance beam generation for future plasma-based colliders or light sources.
\end{abstract}

DOI: 10.1103/PhysRevX.10.041015

Subject Areas: Optics, Photonics, Plasma Physics

\section{INTRODUCTION}

Plasma-based high-gradient wakefield accelerators have attracted significant interest in recent years due to their potential for a significant reduction in the size and cost of future accelerators [1]. They seem particularly attractive as drivers for compact high-brightness photon sources [2,3] but may also play a role as building blocks for $\mathrm{TeV}$-scale high-energy physics machines [4]. Important milestones include the generation of monoenergetic electron beams [5-7] and sustained acceleration of more than a $\mathrm{GeV}$

\footnotetext{
*a.doepp@physik.uni-muenchen.de

†stefan.karsch@physik.uni-muenchen.de
}

Published by the American Physical Society under the terms of the Creative Commons Attribution 4.0 International license. Further distribution of this work must maintain attribution to the author(s) and the published article's title, journal citation, and DOI. Open access publication funded by the Max Planck Society.
[8-13], as well as generation and application of spatially coherent, ultrashort $\mathrm{x}$-ray sources based on accelerated particle beams [14-19]. So far, this novel generation of accelerators has been used to drive applications such as $\mathrm{x}$-ray imaging [20-23] and tomography [24-26], as well as femtosecond electron diffraction [27], femtochemistry [28], and ultrafast spectroscopy $[29,30]$.

The technology relies on an intense drive beam, either a laser pulse or a particle bunch, plowing through a plasma medium and pushing the electrons aside by their ponderomotive force or Coulomb repulsion, respectively. This sets up charge separation fields that pull electrons back and cause them to oscillate around their equilibrium position. As the driver travels though the medium with a velocity close to the speed of light, the field structure ("wakefield") follows at the same speed. Electrons injected into this moving wakefield can be accelerated as a so-called witness bunch. The accelerating field structure has a typical length scale given by the plasma wavelength $\left(\lambda_{p}\right)$, and both accelerating and focusing gradients are several orders of 
magnitude larger than in radio-frequency (rf) accelerators, leading to very dense and ultrashort accelerated particle bunches (some tens of femtoseconds duration). Depending on the driver type, the process is called either laser wakefield acceleration (LWFA) [31] or particle wakefield acceleration (PWFA) [32]. While the former can be studied at many high-intensity laser laboratories at drive pulse powers starting at the terawatt level, the latter typically requires drive bunches that can be provided only by a national-laboratory-scale $\mathrm{rf}$ accelerator.

Despite the fact that both types differ in certain details of wakefield excitation, ionization, and propagation [33], they begin to merge in the case of high-power LWFA. The beam-loading effect [34], which describes the dependence of the accelerating fields on the current of the accelerated bunch, can be understood as the onset of a scarcely studied intermediary regime between LWFA and PWFA. This regime offers new possibilities to produce ultralow-emittance beams as needed for next-generation compact free-electron lasers [35,36]. For instance, Manahan et al. [37] show that beam loading caused by a so-called "escort" bunch could flatten the fields experienced by a subsequently injected "witness" beam. Alternatively, Hidding et al. [38] propose to use a high-charge electron beam from LWFA as a "driver" for a subsequent PWFA stage, giving access to schemes providing electrons with ultralow emittance [39-41] in a compact setup.

In LWFA, the wakefield is created by a high-intensity laser pulse [42-45]. Because of the oscillating nature of electromagnetic waves, lasers are relatively inefficient in setting up a charge separation, and a net energy gain of electrons between cycles is possible only by exploiting the gradient of the electromagnetic field. The force responsible for setting up the wakefield is thus the ponderomotive force [42]

$$
\vec{F}_{\text {pond }}=-\frac{m_{e} c^{2}}{2\langle\gamma\rangle} \vec{\nabla}\left\langle\vec{a}^{2}\right\rangle
$$

with $m_{e}$ as the electron mass, $c$ is the speed of light in vacuum, $\langle\gamma\rangle$ is the cycle-averaged Lorentz factor, and $\vec{a}=$ $e \vec{A} /\left(m_{e} c\right)=\vec{E}_{0} /\left(m_{e} c^{2} / e\right) \times\left(\lambda_{0} / 2 \pi\right) \simeq 0.31 \times \vec{E}_{0}\left[\mathrm{TVm}^{-1}\right] \times$ $\lambda_{0}[\mu \mathrm{m}]$ is the laser's local normalized vector potential, where $\lambda_{0}$ denotes the wavelength of the laser pulse and $\vec{E}_{0}$ stands for the electric field strength of the laser.

Just as the ponderomotive force for a laser driver in LWFA, the space charge field of a particle driver in PWFA is unipolar. However, the former is just a second-order force that is proportional to the gradient of a spatially inhomogeneous oscillating field and, therefore, will not be as strong as the peak force of the laser field under typical LWFA conditions. In that sense, a laser is relatively less efficient in exciting a plasma wave. This difference can lead to the somewhat counterintuitive result that for many experimental parameters, despite its lower total energy, the laser-generated electron bunch can drive a similar wakefield as the laser pulse itself.

The relation between the two driver types can be quantified in the linear wakefield regime, where the plasma perturbation introduced by a driving potential is given by [46]

$$
\left(\frac{\partial^{2}}{\partial t^{2}}+\omega_{p}^{2}\right) \frac{\delta n}{n_{0}}=\underbrace{-\omega_{p}^{2} \frac{n_{b}}{n_{0}}}_{\propto \phi_{\mathrm{el}}}+\underbrace{c^{2} \Delta \frac{\vec{a}^{2}}{2}}_{\propto \phi_{\mathrm{pond}}} .
$$

Here, $\omega_{p}$ is the plasma frequency, $n_{b}$ is the electron bunch density, and $\delta n=n_{e}-n_{0}$, with the plasma density $n_{e}$ and $n_{0}$ denotes the background plasma density. The right-hand side of the equation is composed of terms proportional to the electrostatic $\left(\phi_{\mathrm{el}}\right)$ and ponderomotive potential $\left(\phi_{\text {pond }}\right)$. Hence, both the space charge term $n_{b} / n_{0}$ and ponderomotive force term $\Delta \vec{a}^{2} / 2$ can have similar influence on the plasma wave formation with the limiting cases of pure LWFA $\left(n_{b}=0\right)$ and pure PWFA $\left(\vec{a}^{2}=0\right)$. While Eq. (2) is instructive to distinguish the influence of laser and particles on the plasma, it is valid only in the one-dimensional case and for $n_{b} / n_{0} \ll 1$ and $a_{0} \ll 1$, where $a_{0}$ denotes the maximum of the local normalized vector potential. For stronger drivers $\left(a_{0} \gg 1, n_{b} / n_{0} \gg 1\right)$, the wakefield becomes nonlinear and approaches the blow-out regime [47], where electrons form a thin sheath around a near-spherical ion cavity (the so-called "bubble") behind the driver $[48,49]$. Note that beam loading in this regime is actually entirely determined by the beam current, which is proportional to the beam charge for a given bunch duration [50]. Most experiments, however, operate in an intermediary regime between perturbation and blow out, which currently lacks a consistent theoretical description [52]. To nevertheless illustrate the interplay between both laser and particle beams which is central to this work, we use self-consistent particle-in-cell simulations [53]. As shown in Fig. 1, beam loading of electrons in LWFA affects the sheath electron trajectories, which, in turn, reduces the longitudinal electric fields [54]. With decreasing laser intensity, this beam-loaded LWFA transitions into a regime that resembles the pure PWFA regime. Here, we still observe a quasilinear LWFA, but the plasma electron motion is dominated by the electron beam, forming an ion cavity behind the electron bunch.

While being essential to the understanding and improvement of LWFA, there exist only a few experimental results on the transitional regimes indicated in green and orange in Fig. 1. First systematic studies are presented by Rechatin et al. $[55,56]$ and establish the correlation between charge and peak energy of an electron beam as a key signature of beam loading in LWFA. Later experiments using ionization-induced injection furthermore emphasize the impact of beam loading on the amount of charge that can be trapped inside a wakefield for a given laser power [57,58]. It has been proposed to use beam loading to flatten the accelerating fields along the injected electron bunch and, thus, 


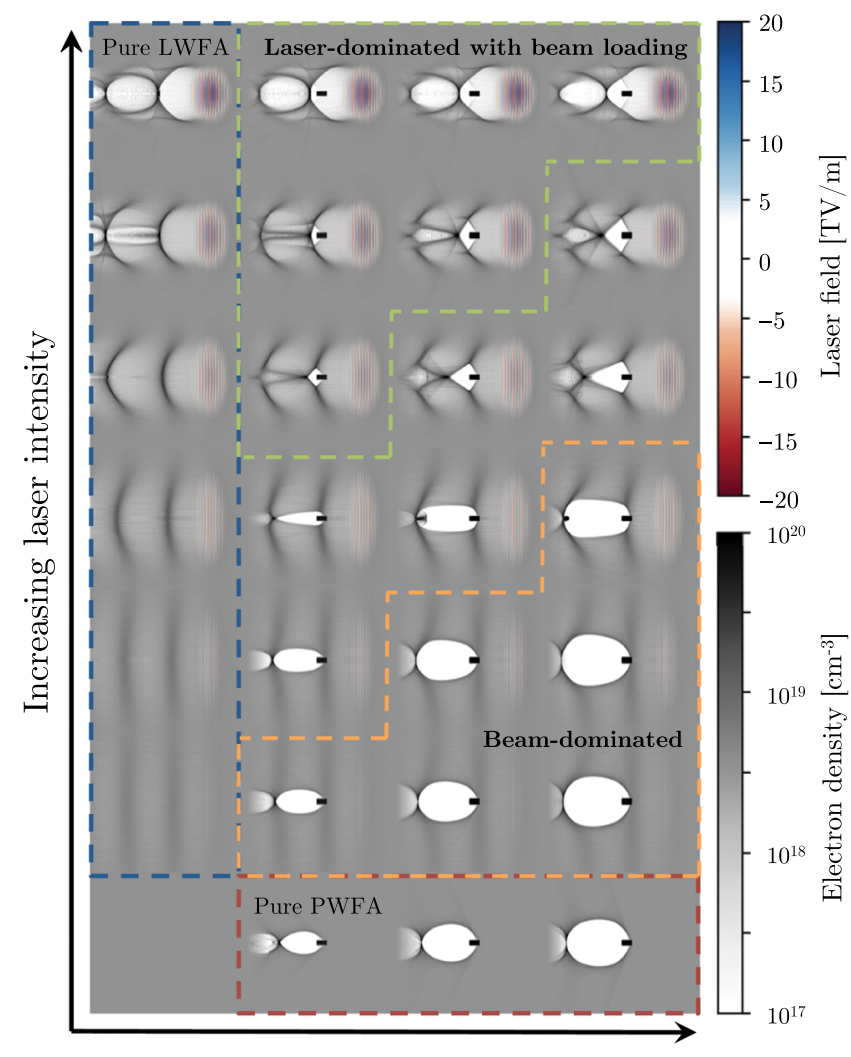

Increasing beam charge

FIG. 1. Schematic view of the different regimes of laser- and beam-driven plasma wakefield acceleration studied in the scope of this work. These plots are based on quasi-3D particle-in-cell simulations, including the transitional regimes from beam-loaded laser wakefields to beam-dominated wakefields with a weaker laser contribution. The laser intensity is varied by changing the focal spot size of a 100-TW laser pulse.

reduce the beam energy spread [59]. While this reduction is mainly applicable in the dephasing-free case of electronbeam-driven PWFAs, first evidence of such an optimal loading regime has been observed in LWFA [58]. Furthermore, indicative signs for a so-called "self-mode transition" [60] from beam-loaded LWFA to pure PWFA have been reported [57,61-64]. In these experiments, the drive laser either depletes in a long plasma target $[61,62,64]$ or diffracts due to ionization defocusing [57], leaving the electron beam as sole driver of the wakefield.

Here, we provide a comprehensive study of wakefield acceleration across different regimes of electron and laser parameters (Secs. II and III). This study is followed by results on beam loading in dual-bunch configurations, which lie at the heart of escort-witness and driver-witness schemes for high-brightness beam generation (Sec. IV). In Sec. V, we present first results on the transition to the beamdominated regime using such dual-energy beams, showing the correlation of the acceleration of a witness beam with the charge of the drive beam. In Sec. VI, we provide an outlook on the scalability of LWFA toward the use of petawatt laser systems. Section VII finally summarizes our findings. Information on simulations and additional experimental data are given in Supplemental Material [51].

\section{HIGH-CHARGE ELECTRON BEAMS FROM SHOCK-FRONT INJECTION}

The results presented in this study are based on shockfront injection, where a shock in a supersonic gas flow is used to create a sharp density down ramp. The sudden change in plasma density results in a localized injection of electrons into a trapping region of the wakefield structure $[65,66]$. While earlier studies on shock-front injection [67-69] show only moderate beam charges of up to few tens of $\mathrm{pC}$, our recent experiments using the ATLAS-300 laser system provide unprecedented performance regarding the stability, total charge, spectral charge density, and divergence of the accelerated electron beams.

In this experiment, the system provides laser pulses with $2 \mathrm{~J}$ energy at $27 \mathrm{fs}$ duration, corresponding to a peak power of $75 \mathrm{TW}$. The laser pulses are focused by an $f / 25$ off-axis parabolic mirror reaching a peak normalized vector potential in vacuum of $a_{0} \approx 1.8$ at focus. A supersonic hydrogen gas jet [70] with a shock front is placed at the laser focal spot position. The shock front is created by the sharp edge of a silicon wafer which projects into the gas jet (cf. Fig. S4 in Supplemental Material [51]). The spectral distribution of the laser-accelerated electron bunch is characterized using a magnet spectrometer, and the spectral charge density is measured by an absolutely calibrated scintillating screen [71]. Further details on all experiments are given in Supplemental Material [51] (cf. Sec. II B) including an illustration of the general setup (Fig. S3).

Figure 2 shows 100 consecutive electron spectra using the shock-front injector operated at a plateau plasma density of $n_{0}=3.0 \times 10^{18} \mathrm{~cm}^{-3}$. The mean charge within the peak is $338 \mathrm{pC}$ and fluctuates by $11 \%$ ( $36 \mathrm{pC} \mathrm{rms)} \mathrm{at} \mathrm{a}$ mean peak energy of $216 \mathrm{MeV}$ with $4 \%$ shot-to-shot s.d. with a rms divergence of $(0.36 \pm 0.08) \mathrm{mrad}$. The peak charge is defined as the integrated charge density around the peak above a certain threshold relative to the maximum charge density. This threshold is often set to 0.5 , corresponding to the full width at half maximum (FWHM). However, to study beam-plasma interaction, we need a measure that reflects the whole bunch charge more accurately. We therefore use $20 \%$ of the peak charge density as the threshold unless specified otherwise. This definition contains most of the peak charge while omitting the lowcharge "dark current" (see, for instance, Fig. 6).

The FWHM absolute energy spread is $(33.0 \pm 7.2) \mathrm{MeV}$ (rms), corresponding to a relative energy spread of $15 \%$. The maximal spectral charge density is (11.7 \pm 1.4) $\mathrm{pC} \mathrm{MeV}^{-1}$, with up to $17 \mathrm{pC} \mathrm{MeV}^{-1}$ for some shots (corresponding to peak angular charge densities of more than $15 \mathrm{pC} \mathrm{MeV}^{-1} \mu \mathrm{sr}^{-1}$ within the angular FWHM of the beam). Note that the individual shots in Fig. 2(a) have a 

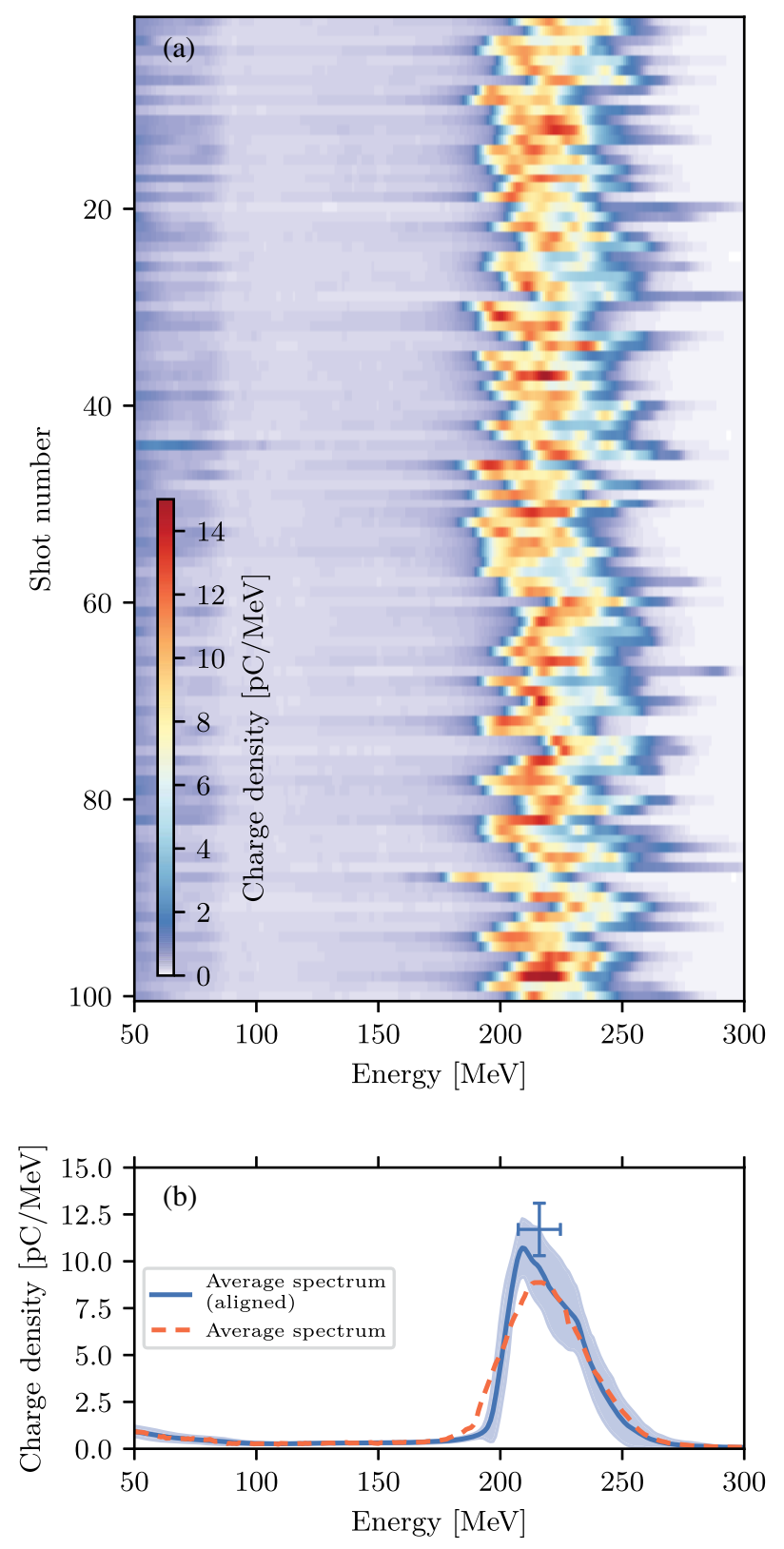

FIG. 2. Optimal performance of the shock-front injector with a 75-TW laser (a quantitative analysis is given in the text below). (a) Angle-integrated electron spectra of 100 consecutive shots. (b) Average spectrum with and without alignment of the beams according to their central energy. The error bar marks the fluctuations in peak spectral charge density and energy.

distinct spectral shape, with many of them being skewed toward lower energy. These features are lost when calculating the average spectrum, since shot-to-shot fluctuations naturally lead to a normally distributed average. We have, therefore, realigned all the spectra according to their central energy (determined via a Gaussian fit) [72] to preserve such features as depicted in Fig. 2(b).

Our results thus show that the amount of charge in the spectral peak in shock-front injection can be comparable to the total charge in self-injection [26], with the further benefit of a clearly localized injection position. Given the known length of the gas target, this benefit permits one to calculate the average accelerating fields and makes this configuration attractive for quantifying the effects of beam loading. However, although the stability of the dataset shown in Fig. 2 can be considered excellent for LWFAs, it turns into a disadvantage when studying beam loading, as it covers only a small range in charge. This result is why, in the following, we deliberately concentrate on data with higher shot-to-shot charge variations, which we attribute to a less stable laser performance.

\section{EFFECTS OF BEAM LOADING ON THE ENERGY SPECTRUM}

In this section, we discuss signatures of beam loading on the spectral shape of laser-accelerated electron beams, followed by a generalization of the results using scaling theory, which is supported by experimental data from a few-cycle laser. We begin our analysis with a dataset containing 100 consecutive shots for shock-front injection at a plateau plasma density of $3.5 \times 10^{18} \mathrm{~cm}^{-3}$. The beam spectra, binned by the peak charge, are shown in Fig. 3(c). On average, these shots contain $(123 \pm 35)$ pC charge within the peak (28.5\% relative charge fluctuation), spanning a range from approximately 60 to $180 \mathrm{pC}$. While the average peak energy is $(232 \pm 30) \mathrm{MeV}$, we observe that the peak energy $E_{\text {peak }}$ is clearly correlated to the beam charge $Q$ :

$E_{\text {peak }}($ ATLAS - 300)

$$
\approx(358 \pm 35) \mathrm{MeV}-(1.01 \pm 0.06) \mathrm{MeV} \times Q[\mathrm{pC}] .
$$

Additional to the reduction in beam energy at higher charges, which is a well-studied effect of beam loading [56,58], we also observe a distinctive spectral shape for beams of different charge; cf. Fig. 3(d). More specifically, there is a sharper cutoff on the low-energy side of the peak, while the spectrum gradually rolls off at the high-energy side, leading to an asymmetrically distributed charge density around the peak. This effect is more pronounced for high-charge bunches and similar to the average spectrum shown in Fig. 2(b). In contrast, low-charge shots show a more symmetric spectrum and exhibit a quasi-Gaussian energy distribution.

To understand how the accelerated electron bunch loads the wake and affects its energy spectrum, the interaction process is extensively simulated for parameters matching the ATLAS-300 experiment using the quasi-3D particle-incell code FBPIC [73]. To estimate the influence of different effects that could cause the observed charge fluctuations, various scans of the laser focus and power, as well as 


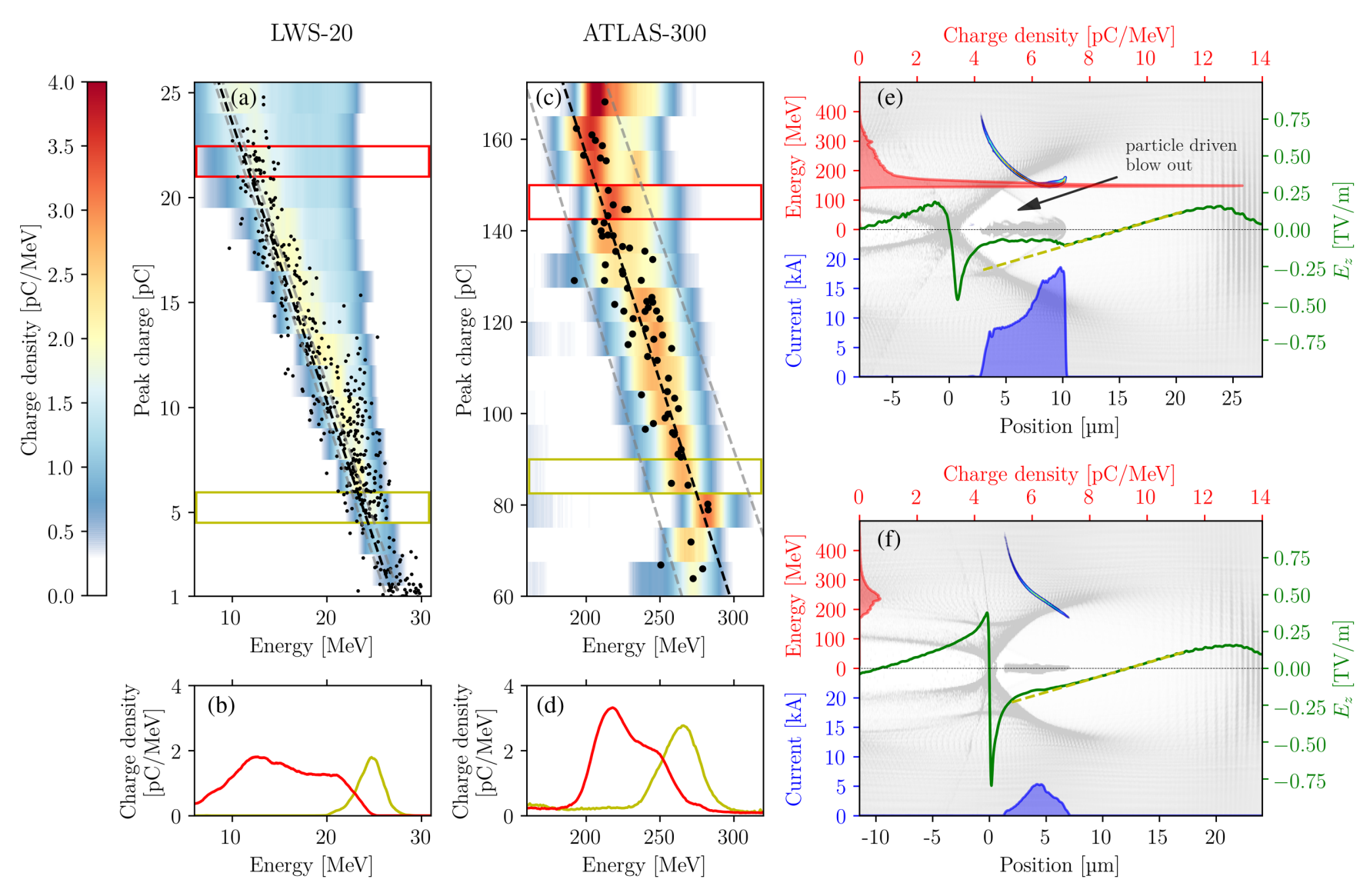

FIG. 3. Beam-loading effects on single laser-accelerated electron bunches. Change of the spectral shape with beam charge. (a) Binned LWS-20 data including the location of the peak energy and peak charge for the individual shots. Lineouts for two bins of high and low charge as marked in their respective colors in (a) are given in (b). (c) shows binned spectra of a dataset acquired using ATLAS-300. Two individual lineouts are given in (d). Dashed black and gray lines indicate linear fits and standard deviations according to Eq. (4) and (3), respectively. (e) and (f) summarize the results of particle-in-cell simulations for the ATLAS-300 parameters for different focus positions, yielding either high beam charge (e) or low beam charge (f). The on-axis field is plotted in green, with dashed yellow lines as the linear fit to the front of the wakefield to show the influence of beam loading. The beam current is plotted as a shaded blue area (bottom), while the spectrum is shown on the left (shaded red). The gray background shows a map of the electron density, while the colored plot shows the electron phase space.

different shock heights and widths, are performed (see Supplemental Material [51] for more details).

The analysis of these simulations reveals that a change in the injected charge indeed leads to a variation of the bunch spectrum due to beam loading which is similar to the experiment. In particular, the simulations show significant changes of both beam charge and energy for shifts of the focus position, as depicted exemplarily in Figs. 3(e) and 3(f). The sensitivity to the focus position is due to the process of shock-front injection, which is closely related to the intensity of the laser at the down ramp. This sensitivity is because the area in the phase space where charge can be trapped depends on the radius $r$ of the ion cavity ("bubble radius"), which scales as $r \propto \sqrt{a_{0} / n_{e}}$; see Ref. [74]. Hence, the normalized laser intensity directly influences the injection process and the involved accelerating fields. Notably, as shown in Fig. S10 [51], the injection is even sensitive to focus position shifts on a scale of $0.2 \mathrm{~mm}$. This scale is well below the Rayleigh length of $1.1 \mathrm{~mm}$, and the equivalent amount of global wavefront curvature in the near field is $\Delta \lambda \lesssim \lambda / 10$. For a large beam and long beam path as in ATLAS, these kind of shifts can easily result from shotto-shot wavefront fluctuations, and the results thus highlight the need of wavefront stabilization to increase the stability of this type of injector.

The simulations explain the observed asymmetry of the electron spectrum by the positive chirp of the electron phase space characteristic for localized injection mechanisms such as shock-front injection. Here, the chirp of the bunch is determined by the variation of the longitudinal field across the wakefield phase, because the bunch is injected into different phase positions at nearly the same time. This positive chirp approximately maps the longitudinal current profile to the energy axis [cf. Fig. 3(f)], even though it is not completely linear. In the case of severe beam loading, the chirp toward the front of the bunch 
vanishes or even reverses. This result leads to a strong peak in energy space and, in consequence, to the skewed spectrum we observe in the experiment, as indicated in Fig. 3(e). Hence, the signature of beam loading resulting in a phase space chirp is a skewed energy spectrum (more details and a quantitative analysis of the skewness for each individual spectrum in Fig. 3 can be found in Supplemental Material [51]).

As beam-loading effects increase with the total charge contained in the accelerating cavity, we expect the asymmetry to become more pronounced for higher total beam charges, which clearly matches the measured data. Indeed, for the ATLAS-300 data, the calculated skewness for almost each electron spectrum is positive and increases with charge, in accordance with simulations. The positive skewness is a typical feature of beam loading in shock-front injection. Our simulations suggest that its dependence on the injected charge can be used as an indicator for the onset of beam loading. It is important to notice that this signature gets easily lost when a beam with large divergence and a nonimaging spectrometer is used or if spectra from multiple shots are averaged without sorting them by charge. Future studies including current measurements with coherent transition radiation (CTR) diagnostics [63] may even allow for quantitative measurements of beam loading or estimation of the electron beam phase space, provided the time ambiguity of the CTR measurement can be resolved.

So far, we have restricted our analysis to very specific experimental parameters. However, it is an interesting feature of plasma wakefields that they are scaling invariant [75], and results from an experiment performed at a plasma density $n$ can be converted to another density $n^{\prime}$ using a scaling parameter $\alpha=\left(n / n^{\prime}\right)^{1 / 2}=\left(\lambda_{p}^{\prime} / \lambda_{p}\right)$. This feature is true not only for PWFA [76], but also for laser wakefields. Here, the driver pulse duration $\left(\tau^{\prime}=\alpha \tau\right)$ and waist $\left(w_{0}^{\prime}=\alpha w_{0}\right)$ scale with $\alpha$, while the peak potential $a_{0}$ is invariant [77], as exemplified by the demonstration of quasimonoenergetic electron acceleration with $\mathrm{mJ}$, few-cycle pulses (e.g., Schmid et al. [78] and Guénot et al. [79]).

To show that this general scaling behavior also extends to beam loading, we present an analysis of results from an experiment performed at almost an order of magnitude higher plasma density, i.e., at a plateau density of approximately $3.1 \times 10^{19} \mathrm{~cm}^{-3}$. The scaling factor $\alpha=0.33$ requires much shorter pulse lengths and tighter focusing, which are provided by the LWS-20 laser at the MaxPlanck-Institute for Quantum Optics (MPQ). Both the laser duration ( $8 \mathrm{fs})$ and waist $\left(w_{0} \approx 8 \mu \mathrm{m}\right)$ are in fair accordance with the scaling requirements. Furthermore, the estimated normalized peak potential $a_{0} \approx 2.0$ is close to the ATLAS300 value. The corresponding dataset consists of 490 electron beam spectra.

Indeed, the experimental results shown in Figs. 3(a) and 3(b) show a very similar evolution of the spectral shape with increasing charge. Furthermore, as for ATLAS data, we measure a correlation between peak energy and charge, in this case

$$
\begin{aligned}
& E_{\text {peak }}(\mathrm{LWS}-20) \\
& \approx(29.0 \pm 0.1) \mathrm{MeV}-(0.84 \pm 0.01) \mathrm{MeV} \times Q[\mathrm{pC}]
\end{aligned}
$$

Notably, the beam energies are much lower than those observed with ATLAS-300. This difference stems from the fact that the LWS-20 experiment deviates from the ideal scaling by using a similar central wavelength as ATLAS300 instead of $\lambda_{0}^{\prime}=\alpha \lambda_{0}$. As a result of this deviation, the ratio between the plasma density and critical density is different in both experiments, leading to faster dephasing in the case of LWS-20 and a lower overall energy gain $\Delta E^{\prime}=\alpha^{2} \Delta E$. The average peak energy of LWS-20 is $(21 \pm 6.4) \mathrm{MeV}$, leading to $\Delta E^{\prime} / \Delta E=0.09 \pm 0.03$, which is close to the expected value of $\alpha^{2}=0.11$. While this remarkable agreement is quantitative, other properties show only qualitative agreement. In order to maintain the beam current, the scaling laws require a scaled charge $Q^{\prime}=\alpha Q$. In the case of LWS-20, we do observe an average charge of $(10.5 \pm 6.0) \mathrm{pC}$, corresponding to $Q^{\prime} / Q=0.09 \pm 0.05$, which is a factor of 3.5 lower than the expectation. Such deviations are not entirely unexpected, as both experiments are only approximately similar, and, for instance, the lack of wavelength adjustment also changes the laser propagation and with it the laser wakefield formation. Nonetheless, the data provide good experimental evidence for scale invariance of laser-driven wakefields.

The beam-loading features observed in this work can, therefore, be generalized to different experimental conditions. While the exact parameters may vary, a similar behavior regarding the spectral shape is expected in all kinds of laser wakefield accelerators with localized injection after entering beam loading, be they driven by fewcycle or petawatt lasers. Furthermore, both experiments exhibit strong charge-energy correlations [Eqs. (3) and (4)], which shows that the electron peak energy fluctuations are dominated by a charge-dependent component. This observation emphasizes the importance of reducing charge fluctuations in LWFA, as shot-to-shot variations of the beam charge will directly translate into peak energy fluctuations. The latter are particularly detrimental to applications relying on chromatic beam transport elements, such as free-electron lasers [80]. Given the sensitivity of shock-front injection on the laser intensity at the density transition, this reduction will likely require the development of active wavefront stabilization.

\section{BEAM LOADING WITH DUAL-ENERGY ELECTRON BEAMS}

So far, we have considered how a high-current electron beam modifies its own accelerating field and, thus, its final 
energy spectrum. However, such a beam will not only affect its direct vicinity, but also modify the wakefield formation in its trail. Here, we discuss how beam loading of a first injected electron beam affects secondary electron beams. As discussed in the introduction, this kind of scenario is particularly promising due to various proposed advanced injection schemes relying on similar conditions. In the following, we discuss two different schemes used for injection of multiple electron beams, namely, combined shock-front [81] and colliding-pulse injection [82] into the same wakefield cavity [83], as well as shock-front injection into subsequent wakefield periods [84]. Because of the different location of the bunches in both schemes (intracavity versus intercavity), these methods are complementary and can be used as a basis for studying escort-witness and driver-witness configurations.

In the first case, we use a gas jet with shock-front injection and split off a part of the main laser beam to obtain a second pulse propagating in the opposite direction and containing an energy of $0.3 \mathrm{~J}$. This counterpropagating pulse is then focused onto the gas target, reaching a normalized peak intensity of up to $a_{1} \simeq 0.9$. When this colliding pulse is active, we can inject a second bunch with lower energy and charge into the same wakefield period via optical injection. Both bunches' peak energies are plotted in Fig. 4. As in the previous section, the energy of the first bunch depends on its own charge $Q_{1}\left[E_{1} \approx(387 \pm 4) \mathrm{MeV}-\right.$ $\left.(1.3 \pm 0.1) \mathrm{MeV} \times Q_{1}[\mathrm{pC}]\right]$. In addition, we now observe a similar trend for the optically injected beam whose energy also correlates with the charge $Q_{1}$ of the first bunch $\left[E_{2} \approx\right.$ $\left.(175 \pm 5) \mathrm{MeV}-(0.7 \pm 0.1) \mathrm{MeV} \times Q_{1}[\mathrm{pC}]\right]$; cf. Fig. 4(a) In contrast, no statistically significant correlation between the charge of the second bunch $Q_{2}$ and the energy of either bunch $E_{1}$ or $E_{2}$ is found as can be seen if we now sort the dataset from Fig. 4(a) by the second bunch's charge [cf. Fig. 4(b)]. The first bunch, therefore, clearly influences the second bunch, which in this case sits in the same plasma period. Nevertheless, the data show that beam-loading effects of the trailing low-energy bunch on itself are negligible.
Shock and optical injection
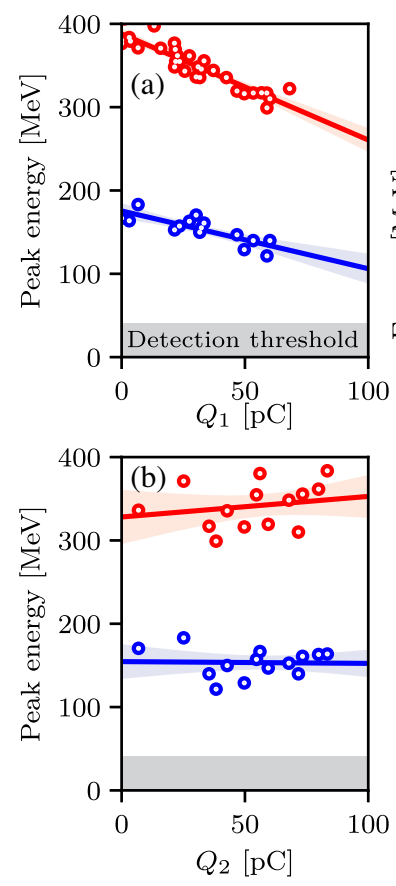
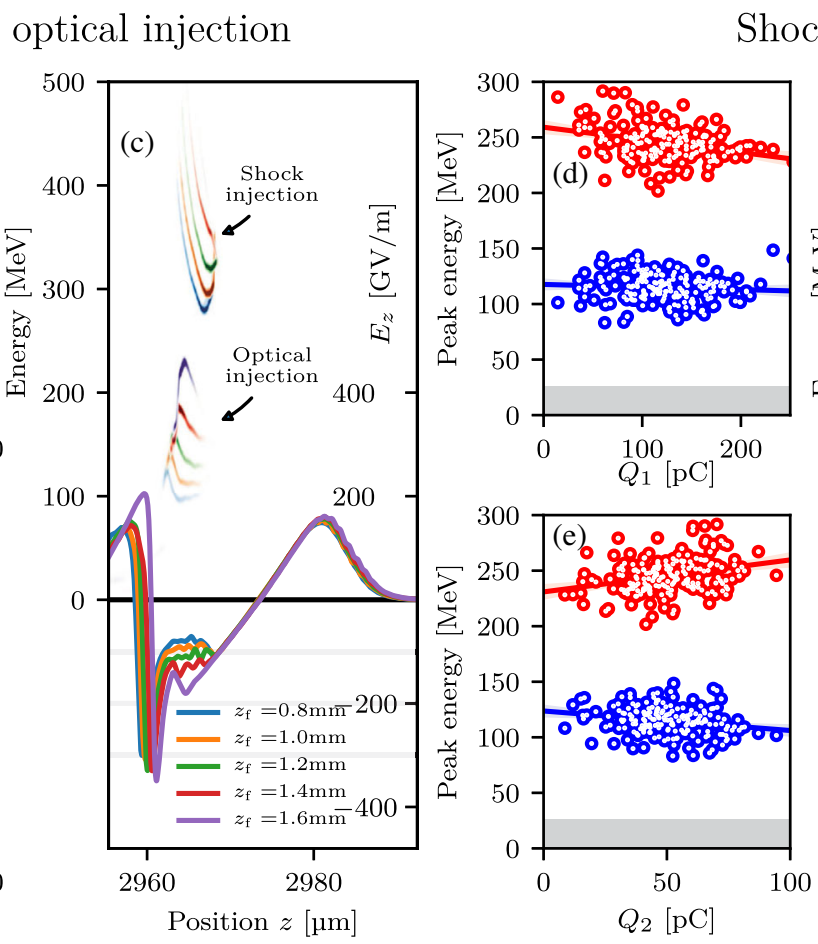

Shock injection

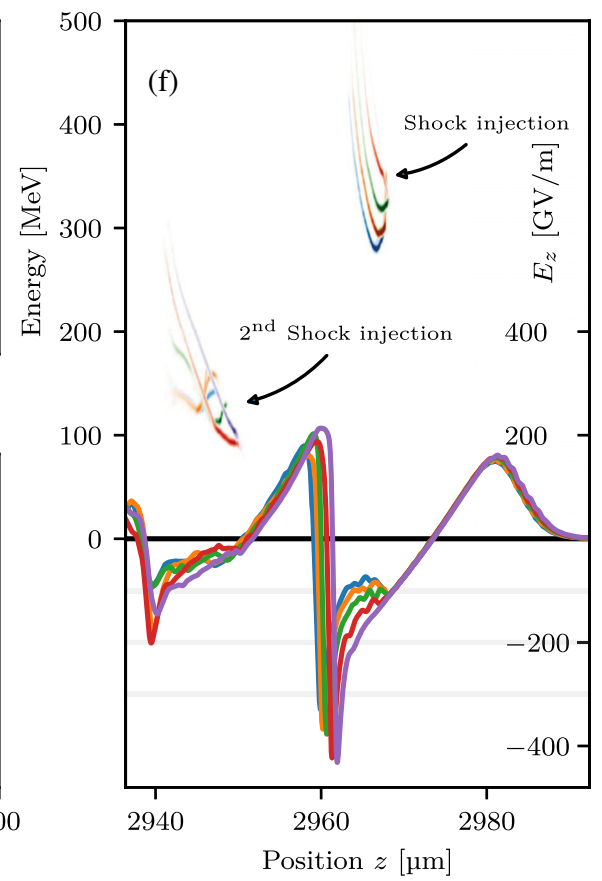

FIG. 4. Effect of beam loading on dual-energy electron beams injected into either the first or second wakefield cavity. First column: Experimental data for electron injection into the first wakefield period via shock and optical injection. The data are sorted either by the charge in the high-energy beam (denoted with charge $\left.Q_{1}\right)$ (a) or by the charge in the low-energy beam $\left(Q_{2}\right)$ (b). Dotted lines show linear fits, and the shaded areas indicate the corresponding confidence interval. The data show a clear correlation between the charge of the shock-injected beam $Q_{1}$ with the energy of the optically injected beam $E_{2}$ (a). (c) Results from particle-in-cell simulations showing both the phase space and the longitudinal on-axis fields. Beams of different charge are injected by changing the absolute focus position $z_{f}$ relative to the density transition $z_{\text {shock }}=0.8 \mathrm{~mm}$. Higher charges $Q_{1}$ decrease the accelerating fields in the wakefield via beam loading, leading to a lower energy of the trailing optically injected electron bunch. Third column: Experimental data for electron injection into the first and second wakefield periods via shock injection. The high-energy beam seems to have no influence on the particle energies in the second wakefield period (d). In contrast to (b), the charge of the second bunch $\left(Q_{2}\right)$ is correlated to the energy of the first electron bunch (e). (f) Particle-in-cell simulations with the same color coding as in the aforementioned plot, showing the same behavior as in (d). The energy of the second beam is uncorrelated to the charge in the first beam, which matches the experimental data. 
In the second experiment, we just use a single laser pulse with shock-front injection but optimize the laser and target parameters (focus and shock position, etc.) to yield a second beam at lower energies. Such trailing beams have already been observed previously (see, for instance, Refs. [81,83]) but usually with a much lower charge than the main beam. These trailing beams stem from shock injection into the second plasma period and are, therefore, intrinsically separated from the leading beam. This bunch distance is approximately given by the plasma wavelength $\lambda_{p}$. Again, the energy of the leading beam correlates with its own charge, even though weaker than in the previous case $\left[E_{1} \approx(259 \pm 3) \mathrm{MeV}-(0.11 \pm 0.03) \mathrm{MeV} \times Q_{1}[\mathrm{pC}]\right]$, because the target parameters are slightly different from the previous experiment. However, in contrast to previous studies [56], the second beam shows no correlation beyond the statistical error $\left[E_{2} \approx(113 \pm 3) \mathrm{MeV}-(0.02 \pm 0.02) \mathrm{MeV} \times\right.$ $Q_{1}$ [pC]] [Fig. 4(d)]. Furthermore, we now observe that the energy of the second beam correlates with its own charge $\left[E_{2} \approx(113 \pm 3) \mathrm{MeV}-(0.18 \pm 0.06) \mathrm{MeV} \times Q_{2}[\mathrm{pC}]\right]$ [Fig. 4(e) is again the same dataset as in Fig. 4(d), but here the peak energies are plotted against the charge of the second bunch). Higher energy of the first bunch, corresponding to less beam loading and a higher remaining wakefield, leads to more charge injected at the shock into the second bucket, and a positive correlation between first bunch energy and second bunch charge is established: $E_{1} \approx(231 \pm 4) \mathrm{MeV}+(0.28 \pm 0.07) \mathrm{MeV} \times Q_{2}[\mathrm{pC}]$.

To understand this behavior, we simulate the two scenarios using particle-in-cell simulations. For better comparison, the parameters of these simulations are modeled on the experiment with shock-front injection into two buckets. This way, injection into the second bucket can be switched on and off by adjusting the simulation window length while keeping the evolution of the plasma wave identical. Based on our previous findings, we use the focus position to adjust the injected charge while keeping the wakefields similar. This adjustment results in electron spectra with decreasing charge and increasing energy the further away the focus position lies from the shock at $z_{\text {shock }}=0.8 \mathrm{~mm}$. For colliding-pulse injection, we initialize a second counterpropagating laser beam with $a_{1}=0.5$ at a distance $\Delta z=1.1 \mathrm{~mm}$ behind the shock on the density plateau. The phase space of the electrons injected due to this optical injection process shows a clear dependence on the scanned focus positions $z_{f}$ and, hence, charge of the first beam [cf. Fig. 4(c)]. Looking at the longitudinal onaxis wakefield $E_{z}$, we can identify the cause of this correlation to be the different amount of beam loading induced by the first beam, i.e., the differing amplitude of $E_{z}$ for each case. This difference changes the threshold for optical injection and modifies the bunch charge and energy. This result confirms the hypothesis deduced from the experimental findings, namely, that the second bunch is a direct witness of beam loading induced by the first. The simulations also indicate that the charge injected via colliding-pulse injection is reduced due to beam loading of the first bunch.

For the second case, we use a longer simulation window to observe also injection into the second wakefield period [cf. Fig. 4(f)]. As in the experiment, the energy of the second injected bunch does not seem to be determined by the charge and beam loading induced by the first bunch, even though it clearly perturbs the first wakefield. In fact, for all different cases, the slope of the wakefield in the second period appears to be nearly independent of beam loading from the first bunch and changes only due to beam loading of the second bunch itself [explaining the correlation $E_{2} \mid Q_{2}$; cf. Fig. 4(f)].

The simulations also help us to understand the $E_{1} \mid Q_{2}$ correlation, which is actually caused by a relation between $Q_{1} \mid Q_{2}$ and $Q_{1} \mid E_{1}$ : During any down-ramp injection process, electrons are more likely to be injected into subsequent wakefield periods due to the progressively slower phase velocity. This result means that, as fewer electrons are captured by the first wakefield period, more electrons can be trapped in the second period and vice versa. We have already established how the charge of the first beam affects its energy, hence explaining the observed correlation.

In accordance with quasi-3D particle-in-cell (PIC) simulations, our results therefore demonstrate how charge and energy of multiple electron bunches are correlated. In particular, our experimental measurements on bunches in subsequent wakefield periods show an unexpectedly weak correlation between the charge of the first bunch and the energy of the second, an effect which could be reproduced in PIC simulations for our conditions. In future experiments, this method could be extended to measure energy and longitudinal charge distributions of multiple pulses as a function of, e.g., the acceleration distance to probe local fields and effects of beam loading across subsequent wakefield cavities and, hence, yield quantitative properties of laser wakefields under different conditions.

Moreover, optical injection can provide an electron beam that witnesses the effect of self-fields of another beam inside the same cavity. This configuration can be used for producing a witness-escort pair as proposed by Manahan et al. [37] in the context of PWFA. Here, the shock-injected beam could potentially act as an escort and flatten the LWFA field that is experienced by an independently injected witness bunch in order to reduce its energy spread. Indeed, this effect is clearly visible in the simulations. For instance, if the laser is focused onto the shock at $z_{f}=0.8 \mathrm{~mm}$, shown in Fig. 4(c), a highercharge $Q_{1}$ is injected, which leads to smaller energy spread in the phase space of the optically injected bunch. So far, the experimental data are not conclusive about this effect, but it is planned to study this configuration further in future campaigns. 


\section{TRANSITION TO THE BEAM-DOMINATED REGIME}

In the preceding sections, we establish that shockinjected electron beams have a sufficiently high charge density to modify the electric fields of the laser wakefield and, thus, influence both their own spectrum and the spectrum of other beams. The underlying force is fundamentally the same that is responsible for the excitation of plasma wakefields, and, as visible in Fig. 1, even in the laser-dominated regime the electron beam generates its own wake, i.e., expelling any electrons that are not blown out from the bubble by the ponderomotive force of the laser. As the laser gets weaker in intensity, e.g., due to energy depletion or diffraction, the electron beam becomes the dominant driver of the wakefield; see the lower part of Fig. 1. Indeed, it was recently observed that a lasergenerated electron beam can drive plasma waves in a pure PWFA regime [76].

While this effect can occur naturally in form of a selfmode transition, it is not easy to control and offers little insight into this transition process. The most systematic studies in this regard use gas targets of variable lengths $[63,64]$, but their interpretation relies heavily on simulations, because the laser intensity cannot be controlled independently of the electron beam energy in this case. In particular, due to self-guiding of the laser pulse, the selfmode transition occurs close to laser depletion at a distance
$L_{\text {depl }} \sim\left(n_{\text {cr }} / n_{e}\right) c \tau$, with $c \tau$ being the pulse length [74]. For resonant wakefield excitation, this value is close to the dephasing length $L_{\mathrm{deph}} \sim\left(n_{\mathrm{cr}} / n_{e}\right)(2 / 3) R_{\text {bubble }}$, where $R_{\text {bubble }}$ is the bubble radius [74]. This result means that the first electron beam also loses energy due to dephasing, which is both inefficient and makes it difficult to distinguish the LWFA- and PWFA-dominated cases in this situation.

To circumvent these problems, we follow another approach here. Instead of extending the gas target toward self-mode transition, we use the different divergences of both the electron and laser beam, i.e., $\theta_{\text {elec }} \sim 1 \mathrm{mrad}$ for the former versus $\theta_{\text {laser }} \gtrsim 40 \mathrm{mrad}$ for the latter [85], to strongly reduce the laser intensity after a vacuum gap. We can then place a second gas target behind the first one such that the electron beam takes over as the primary wakefield driver. Importantly, in this setup the electron beam energy remains unchanged during the transition from the beam-loaded LWFA to the beam-dominated regime in the second stage.

A distinctive sign for the transition to the beamdominated regime is that a second witness bunch with the correct delay experiences a positive correlation of its energy with the first bunch's charge due to the increasing wakefield amplitude induced by a higher driver bunch charge. As discussed before, dual-bunch shock injection intrinsically provides a driver-witness pair with a bunch separation of approximately $\lambda_{p}$. The leading $200-300 \mathrm{MeV}$ bunch serves as driver of the wakefield in the second
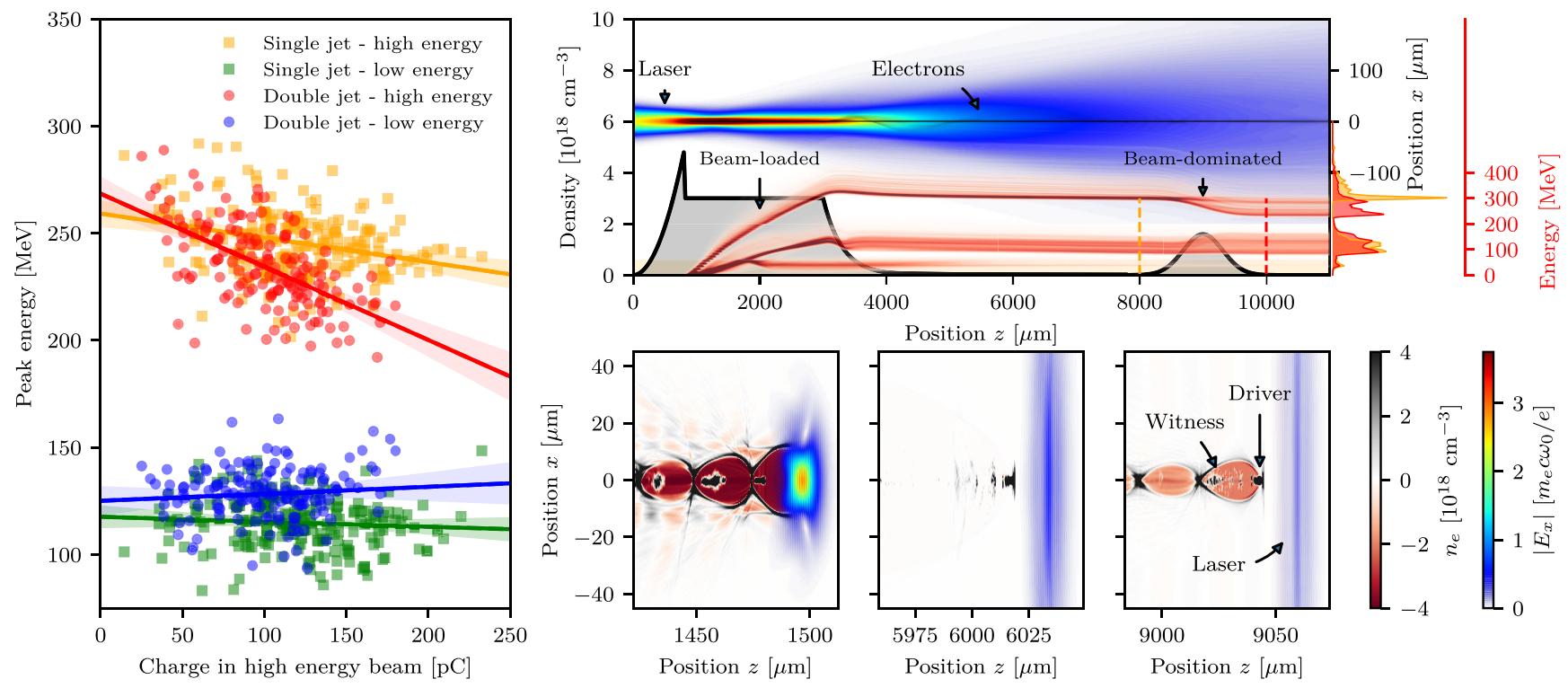

FIG. 5. Electron acceleration in the beam-dominated regime. Left: Energy from the driving electron bunch (high energy) is transferred to the witness bunch (low energy) in the second stage. The higher the drive bunch charge, the higher the witness energy gain. The driver itself, therefore, loses energy and charge. Top right: Results of a start-to-end simulation showing both the laser and beam evolution (upper part) as well as the plasma density (gray, lower part) and energy spectrum at each position (red). The leading electron beam contains a charge of $105 \mathrm{pC}$. It gets decelerated in the second jet, while a part of the second bunch is accelerated, just as observed in the experiment. The energy spectrum before the jet at $z=8 \mathrm{~mm}$ and after the jet at $z=10 \mathrm{~mm}$ is shown on the right. Bottom right: Snapshots for three different positions, i.e., inside the laser wakefield accelerator, during propagation in the near vacuum between both jets, and inside the beam-dominated second jet. The respective plasma densities and electric fields are color coded. 
nozzle, whereas the subsequent $75-125 \mathrm{MeV}$ bunch probes these fields. Please recall that the energy of the low-energy beam is essentially independent of the charge of the high-energy beam in this case. We interferometrically measure a peak plasma density of $1.4 \times 10^{18} \mathrm{~cm}^{-3}$ (i.e., $\rho \simeq 0.225 \mathrm{pC} \mu \mathrm{m}^{-3}$ ) in the second plasma from a nozzle with an opening diameter of $1 \mathrm{~mm}$. For this density, we expect the second bunch to be situated in the accelerating phase of the beam-dominated wakefield.

As presented in Fig. 5, the beam parameters are significantly altered by the second stage. Similar to Chou et al. [86], we also observe that the average beam charge is reduced by about $20 \%$ and the high-energy beam is decelerated. While this observation alone could be explained with dephasing in a laser-driven wakefield, we also observe a charge-dependent acceleration of the witness by $10-20 \mathrm{MeV}$, translating into an acceleration gradient of $10-20 \mathrm{GeV} \mathrm{m}^{-1}$ (see Fig. 5). As mentioned earlier, this result is the opposite of what would be expected in the laser-dominated case.

We simulate the entire target setup in start-to-end simulations using a boosted frame [87,88]. The results are depicted in Fig. 5. According to simulations, the normalized vector potential of the laser drops from $a_{0} \simeq$ 3.2 at the exit of the first gas jet to below $a_{0}<0.6$ when entering the second jet due to diffraction. There is no beam collapse due to self-focusing in the second jet, and, hence, the laser drives only a weak linear plasma wave.

In contrast, the small divergence of approximately $1 \mathrm{mrad}$ keeps the density of the electron bunch high and allows them to become the primary driving force of the wakefield. The simulations clearly show deceleration of the highenergy bunch in the second jet, combined with acceleration of the low-energy particle beam. As in the experiment, this effect depends on the charge of the first beam. In simulations, we mimic this effect by adjusting the focus position (cf. Sec. IV), where the case displayed in Fig. 5 corresponds to a focus at $z_{f}=1.0 \mathrm{~mm}$ with a total charge of $105 \mathrm{pC}$ in the driver. Here, the observed acceleration and deceleration is comparable to the experiment. An example for the case with higher charge in the driver $(125 \mathrm{pC})$ is given in Supplemental Material [51]. In this scenario, the spectra of high- and low-energy beams almost overlap after leaving the second gas target.

While the electron beam clearly dominates the wakefield formation, the head of the bunch still experiences the linear wakefield driven by the laser. With appropriate timing, a weak laser pulse could therefore, for instance, be used to mitigate head erosion in laboratory-scale PWFA experiments. Expanding on this idea, the laser can also be used for guiding of the electron beam in a configuration similar to a laser-plasma lens [89], and some aspects of the setup could be analyzed with models developed in this context [90]. In the limit of negligible laser intensity, this setup becomes equivalent to a compact externally seeded PWFA stage such as presented by Kurz et al. [84]. However, even for low laser intensity, the configuration discussed here has the advantage that no plasma mirror is necessary to block the laser and that the laser preionizes the plasma.

\section{TOWARD JOULE-CLASS ELECTRON BEAMS}

The results presented so far are obtained with sub-100TW lasers. With higher laser power, the accelerated charge is also increasing, and the energy contained in the relativistic electron beam is approaching the joule energy level.

To quantify the scaling behavior, we perform shock-front injection with various laser energies and a pulse duration of approximately $27 \mathrm{fs}$. In a first scaling test from 10 to $70 \mathrm{TW}$, we find that the charge in the peak follows an approximately linear trend:

$$
Q_{\text {peak }} \approx 5.5 \mathrm{pC} \times\left(P[\mathrm{TW}]-P_{\text {inj }}[\mathrm{TW}]\right),
$$

where $P_{\text {inj }}=15 \mathrm{TW}$ is the power threshold for injection. The laser power in these experiments is adjusted by detuning the pump lasers and is generally limited by the damage threshold of the compressor gratings to below 70 TW. Nevertheless, in a separate campaign, a few shots are taken with 110 TW (3 J) on target. Here, we observe even more charge with up to $1.2 \mathrm{nC}$ in the energy range above $80 \mathrm{MeV}$ and a peak charge of $602 \mathrm{pC}$, corresponding to $50 \%$ of the total charge, at a maximal peak charge density of $15 \mathrm{pC} \mathrm{MeV}^{-1}$ (see Fig. 6). The FWHM divergence is found to be $3.1 \mathrm{mrad}$. These data are still in agreement with the above linear scaling. The total energy of the electron beam observed on the spectrometer is $288 \mathrm{~mJ}$, corresponding to an energy transfer rate from the laser pulse to the electron beam of almost $10 \%$.

As part of the commissioning of the new ATLAS-3000 multipetawatt laser, we also perform first tests with an energy on target of up to $10 \mathrm{~J}$ and a peak power reaching $330 \mathrm{TW}$ [91]. A typical shot from these experiments is shown in Fig. 6. It also displays the skewed spectral shape established in Sec. III. Over a series of 50 shots, we measure a charge in the peak of $(1.4 \pm 0.2) \mathrm{nC}$, $(1.1 \pm 0.2) \mathrm{nC}$ in FWHM, with an average peak charge density of $(22 \pm 4) \mathrm{pC} \mathrm{MeV}^{-1}$ and an average energy of $(334 \pm 39) \mathrm{MeV}$. The average energy spread is $60 \mathrm{MeV}$ or $18 \%$, respectively. Please note that this experiment is not optimized for high energy, but just high spectral charge density. The measured divergence is $(2.5 \pm 0.7) \mathrm{mrad}$. Assuming a round electron beam, peak angular charge densities of approximately $5 \mathrm{pC} \mathrm{MeV}^{-1} \mu \mathrm{sr}^{-1}$ are estimated within the angular FWHM of the beam. Integrating over the entire spectrum, we measure a total transferred energy to the relativistic electrons of $(0.6 \pm 0.1) \mathrm{J}$. Also, since the scintillation screen is saturated in some shots, these values are lower limits, which may partially explain why the measured beam charge remains slightly below the prediction of the scaling [1.7 nC; cf. Eq. (5)]. 

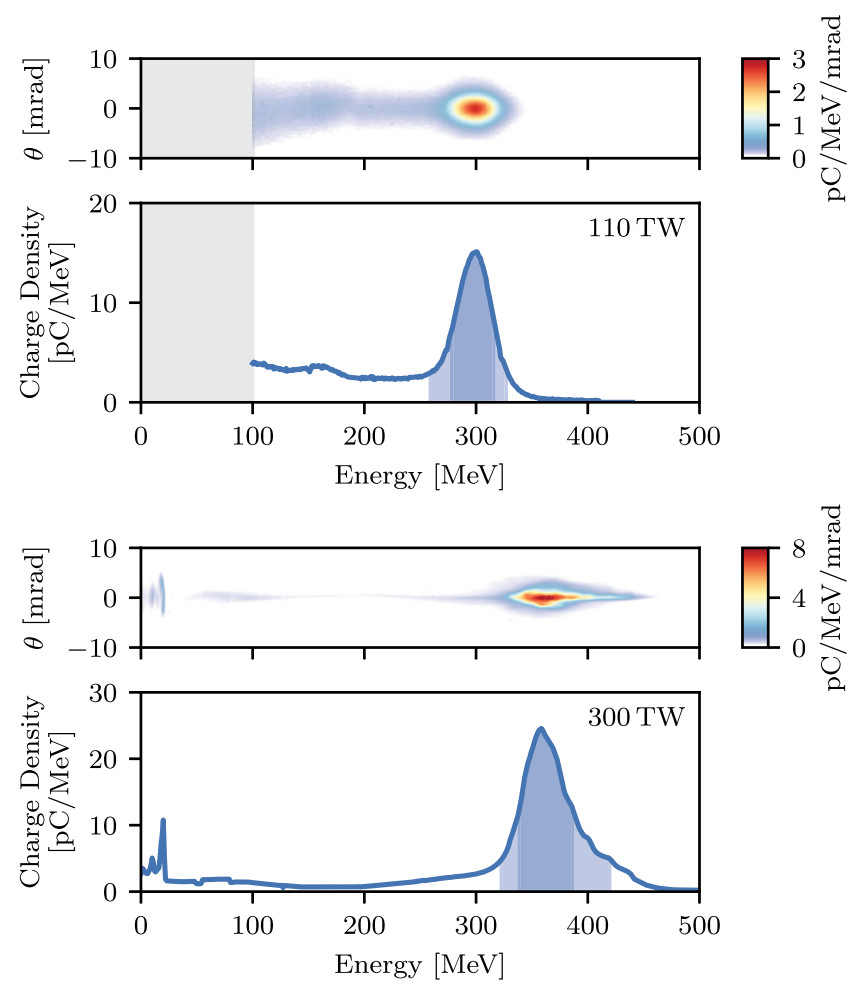

FIG. 6. Electron spectra from shock-front injection, plotted on a linear energy scale. Top: 110-TW case-The total charge above $100 \mathrm{MeV}$ is $1.2 \mathrm{nC}$, and the peak contains $608 \mathrm{pC}$ within the $20 \%$ width (lighter shaded area) and $470 \mathrm{pC}$ within the FWHM interval (darker shaded area). The spectral charge density reaches $15 \mathrm{pC} \mathrm{MeV}^{-1}$. Electrons below $100 \mathrm{MeV}$ are not detected. Bottom: 300-TW case-The total charge above $100 \mathrm{MeV}$ is $1.74 \mathrm{nC}$. The peak contains a charge of $1.3 \mathrm{nC}$ (lighter shaded area), with $0.94 \mathrm{nC}$ in the FWHM (shaded area). The peak charge density exceeds $24 \mathrm{pC} \mathrm{MeV}^{-1}$.

In order to extrapolate toward the petawatt level, we therefore perform PIC simulations for different laser powers. The simulations are in agreement with the experimental data and show a transition from a linear scaling $Q_{\text {peak }} \propto P$ to $Q_{\text {peak }} \propto \sqrt{P}$ for $P \gtrsim 200 \mathrm{TW}$; see Supplemental Fig. S5 [51]. We interpret this behavior as a transition from the partially loaded to the fully loaded laser wakefield regime [57], with the latter having a well-established $\sqrt{P}$ scaling [74]. Based on our analysis, we estimate a peak charge of the order of $2.5 \mathrm{nC}$ and more than $100 \mathrm{kA}$ peak current for a one-petawatt laser driver, which is more than twice the value predicted by the scaling for self-truncated ionization injection [58].

\section{CONCLUSIONS AND OUTLOOK}

We have presented results on high-charge electron beams produced via LWFA, which feature exceptionally high spectral charge densities and low divergence. While the beam parameters are promising for demonstrating a laserbased free-electron laser in the near-optical regime [92], many other applications require even better beam quality in terms of emittance and, more generally, brightness. Because of their high charge density, the laser-accelerated beams can be used to access new regimes of beam-loaded and beam-dominated wakefield acceleration. This intermediate regime of LWFA and PWFA holds promise for the generation of ultralow-emittance beams in a compact setup in the future.

Our analysis clearly shows that we are capable of reaching different regimes of beam loading, evidenced by a dependence of both the final beam energy as well as the spectral shape of laser-accelerated electron beams on the beam charge. This effect is scale invariant and was consistently observed in different regimes of LWFA, i.e., in the few-cycle regime using the 100-mJ-class LWS-20 laser and in the joule-class regime with the ATLAS-300 laser.

When combined with not a single, but two electron bunches, beam loading can become a powerful tool to optimize LWFA. In a proof-of-principle experiment, we have obtained first results on beam loading for electron beams injected into the same wakefield period as well as subsequent wakefields. The former serves as precursor for a escort-witness configuration [37], while the latter was used to study beam-dominated LWFA in a driver-witness configuration [38]. Here, we demonstrated a charge dependence of the witness acceleration in a second wakefield stage, which is a clear signature for the transition to the beam-dominated regime. The observable energy gain in this setup was mainly limited by the energy separation between both bunches, which will be increased in future experiments. Furthermore, bunch duration and separation measurements via coherent transition radiation are expected to yield more quantitative information on both dual-beam configurations.

As an outlook, the scalability of the shock-injection scheme was studied by means of experiments and simulations in the ranges up to $330 \mathrm{TW}$ and $1 \mathrm{PW}$, respectively. Based on these studies it is likely that laser wakefield accelerators driven by petawatt lasers will very soon deliver joule-class electron beams with 100-kA-class currents, which will give access to new regimes of laser-beam interaction and radiation generation.

\section{ACKNOWLEDGMENTS}

We acknowledge K. Schmid and C. M. S. Sears, who performed experiments on the LWS-20 laser at MPQ. This work was supported by the DFG through the Cluster of Excellence Munich-Centre for Advanced Photonics (MAP EXC 158), TR-18 funding schemes and the Max Planck Society. Furthermore, this work has been carried out within the framework of the EUROfusion Consortium and has received funding from the Euratom research and training programme 2014-2018 and 2019-2020 under Grant agreement No. 633053. The authors gratefully acknowledge the Gauss Centre for Supercomputing e.V. for funding this 
project by providing computing time on the GCS Supercomputer SuperMUC at the Leibniz Supercomputing Centre. F. M.F. is part of the Max Planck School of Photonics (MPSP), jointly supported by BMBF, Max Planck Society, and Fraunhofer Society. L. V. acknowledges support from Vetenskapsrådet/Swedish Research Council (2016-05409 and 2019-02376).

J. G. and A. D. contributed equally to this work. A. D., J. G., M. F. G., H. D., S. S., G. S., and S. K. set up and/or performed the experiments with ATLAS-300. F. M.F., A. D., H. D., M. F. G., J. G., and S. K. performed the experiments with ATLAS-3000. A. D. and J. G. analyzed the data from ATLAS-300 and performed simulations. A. B. and L. V. provided complementary data with LWS-20 results. F. M.F. analyzed data from the ATLAS-3000 commissioning experiments. All authors discussed the results. A. D. and J. G. wrote the paper. S. K. supervised the project.

[1] V. Malka, J. Faure, Y. A. Gauduel, E. Lefebvre, A. Rousse, and K. T. Phuoc, Principles and Applications of Compact Laser-Plasma Accelerators, Nat. Phys. 4, 447 (2008).

[2] S. Corde, K. T. Phuoc, G. Lambert, R. Fitour, V. Malka, A. Rousse, A. Beck, and E. Lefebvre, Femtosecond X Rays from Laser-Plasma Accelerators, Rev. Mod. Phys. 85, 1 (2013).

[3] F. Albert and A. G. R. Thomas, Applications of Laser Wakefield Accelerator-Based Light Sources, Plasma Phys. Controlled Fusion 58, 103001 (2016).

[4] W. Leemans and E. Esarey, Laser-Driven Plasma-Wave Electron Accelerators, Phys. Today 62, No. 3, 44 (2009).

[5] J. Faure, Y. Glinec, A. Pukhov, S. Kiselev, S. Gordienko, E. Lefebvre, J. P. Rousseau, F. Burgy, and V. Malka, A LaserPlasma Accelerator Producing Monoenergetic Electron Beams, Nature (London) 431, 541 (2004).

[6] S. P. D. Mangles, C. D. Murphy, Z. Najmudin, A. G. R. Thomas, J. L. Collier, A. E. Dangor, E. J. Divall, P. S. Foster, J. G. Gallacher, C. J. Hooker, D. A. Jaroszynski, A. J. Langley, W. B. Mori, P. A. Norreys, F. S. Tsung, R. Viskup, B. R. Walton, and K. Krushelnick, Monoenergetic Beams of Relativistic Electrons from Intense Laser-Plasma Interactions, Nature (London) 431, 535 (2004).

[7] C. G. R. Geddes, C. Toth, J. van Tilborg, E. Esarey, C. B. Schroeder, D. Bruhwiler, C. Nieter, J. Cary, and W. P. Leemans, High-Quality Electron Beams from a Laser Wakefield Accelerator Using Plasma-Channel Guiding, Nature (London) 431, 538 (2004).

[8] W. P. Leemans, B. Nagler, A. J. Gonsalves, C. Tóth, K. Nakamura, C. G. R. Geddes, E. Esarey, C. B. Schroeder, and S. M. Hooker, GeV Electron Beams from a CentimetreScale Accelerator, Nat. Phys. 2, 696 (2006).

[9] I. Blumenfeld, C. E. Clayton, F. J. Decker, M. J. Hogan, C. K. Huang, R. Ischebeck, R. Iverson, C. Joshi, T. Katsouleas, N. Kirby, W. Lu, K. A. Marsh, W. B. Mori, P. Muggli, E. Oz, R. H. Siemann, D. Walz, and M. M. Zhou, Energy Doubling of $42 \mathrm{GeV}$ Electrons in a Metre-Scale Plasma Wakefield Accelerator, Nature (London) 445, 741 (2007).
[10] X. Wang et al., Quasi-monoenergetic Laser-Plasma Acceleration of Electrons to $2 \mathrm{GeV}$, Nat. Commun. 4, 1988 (2013).

[11] M. Litos et al., High-Efficiency Acceleration of an Electron Beam in a Plasma Wakefield Accelerator, Nature (London) 515, 92 (2014).

[12] E. Adli et al., Acceleration of Electrons in the Plasma Wakefield of a Proton Bunch, Nature (London) 561, 363 (2018).

[13] A. J. Gonsalves et al., Petawatt Laser Guiding and Electron Beam Acceleration to $8 \mathrm{GeV}$ in a Laser-Heated Capillary Discharge Waveguide, Phys. Rev. Lett. 122, 084801 (2019).

[14] A. Rousse, K. T. Phuoc, R. Shah, A. Pukhov, E. Lefebvre, V. Malka, S. Kiselev, F. Burgy, J. P. Rousseau, D. Umstadter, and D. Hulin, Production of a keV X-Ray Beam from Synchrotron Radiation in Relativistic Laser-Plasma Interaction, Phys. Rev. Lett. 93, 135005 (2004).

[15] S. Kneip et al., Bright Spatially Coherent Synchrotron X-Rays from a Table-Top Source, Nat. Phys. 6, 980 (2010).

[16] K. T. Phuoc, S. Corde, C. Thaury, V. Malka, A. Tafzi, J. P. Goddet, R. C. Shah, S. Sebban, and A. Rousse, All-Optical Compton Gamma-Ray Source, Nat. Photonics 6, 308 (2012).

[17] N. D. Powers, I. Ghebregziabher, G. Golovin, C. Liu, S. Chen, S. Banerjee, J. Zhang, and D. P. Umstadter, Quasimonoenergetic and Tunable X-Rays from a Laser-Driven Compton Light Source, Nat. Photonics 8, 28 (2014).

[18] K. Khrennikov, J. Wenz, A. Buck, J. Xu, M. Heigoldt, L. Veisz, and S. Karsch, Tunable All-Optical Quasimonochromatic Thomson X-Ray Source in the Nonlinear Regime, Phys. Rev. Lett. 114, 195003 (2015).

[19] A. Döpp, B. Mahieu, A. Lifschitz, C. Thaury, A. Doche, E. Guillaume, G. Grittani, O. Lundh, M. Hansson, J. Gautier, M. Kozlova, J. P. Goddet, P. Rousseau, A. Tafzi, V. Malka, A. Rousse, S. Corde, and K. T. Phuoc, Stable Femtosecond $X$-Rays with Tunable Polarization from a Laser-Driven Accelerator, Light Sci. Appl. 6, e17086 (2017).

[20] S. Fourmaux, S. Corde, K. T. Phuoc, P. Lassonde, G. Lebrun, S. Payeur, F. Martin, S. Sebban, V. Malka, A. Rousse, and J.C. Kieffer, Single Shot Phase Contrast Imaging Using Laser-Produced Betatron X-Ray Beams, Opt. Lett. 36, 2426 (2011).

[21] S. Kneip, C. McGuffey, F. Dollar, M. S. Bloom, V. Chvykov, G. Kalintchenko, K. Krushelnick, A. Maksimchuk, S. P. D. Mangles, T. Matsuoka, Z. Najmudin, C. A. J. Palmer, J. Schreiber, W. Schumaker, A. G. R. Thomas, and V. Yanovsky, $X$-Ray Phase Contrast Imaging of Biological Specimens with Femtosecond Pulses of Betatron Radiation from a Compact Laser Plasma Wakefield Accelerator, Appl. Phys. Lett. 99, 093701 (2011).

[22] A. E. Hussein et al., Laser-Wakefield Accelerators for HighResolution X-Ray Imaging of Complex Microstructures, Sci. Rep. 9, 3249 (2019).

[23] D. Guénot, K. Svendsen, J. B. Svensson, H. Ekerfelt, A. Persson, O. Lundh, and E. Berrocal, Simultaneous LaserDriven X-Ray and Two-Photon Fluorescence Imaging of Atomizing Sprays, Optica 7, 131 (2020).

[24] J. Wenz, S. Schleede, K. Khrennikov, M. Bech, P. Thibault, M. Heigoldt, F. Pfeiffer, and S. Karsch, Quantitative X-Ray 
Phase-Contrast Microtomography from a Compact LaserDriven Betatron Source, Nat. Commun. 6, 7568 (2015).

[25] J. M. Cole, J. C. Wood, N. C. Lopes, K. Poder, R. L. Abel, S. Alatabi, J. S. J. Bryant, A. Jin, S. Kneip, K. Mecseki, D. R. Symes, S. P. D. Mangles, and Z. Najmudin, LaserWakefield Accelerators as Hard X-Ray Sources for 3D Medical Imaging of Human Bone, Sci. Rep. 5, 13244 (2015).

[26] A. Döpp, L. Hehn, J. Götzfried, J. Wenz, M. Gilljohann, H. Ding, S. Schindler, F. Pfeiffer, and S. Karsch, Quick X-Ray Microtomography Using a Laser-Driven Betatron Source, Optica 5, 199 (2018).

[27] Z. H. He, B. Beaurepaire, J. A. Nees, G. Gallé, S. A. Scott, J. R. S. Pérez, M. G. Lagally, K. Krushelnick, A. G. R. Thomas, and J. Faure, Capturing Structural Dynamics in Crystalline Silicon Using Chirped Electrons from a Laser Wakefield Accelerator, Sci. Rep. 6, 36224 (2016).

[28] Y. A. Gauduel, Y. Glinec, J. P. Rousseau, F. Burgy, and V. Malka, High Energy Radiation Femtochemistry of Water Molecules: Early Electron-Radical Pairs Processes, Eur. Phys. J. D 60, 121 (2010).

[29] B. Mahieu, N. Jourdain, K. T. Phuoc, F. Dorchies, J. P. Goddet, A. Lifschitz, P. Renaudin, and L. Lecherbourg, Probing Warm Dense Matter Using Femtosecond X-Ray Absorption Spectroscopy with a Laser-Produced Betatron Source, Nat. Commun. 9, 3276 (2018).

[30] B. Kettle et al., Single-Shot Multi-keV X-Ray Absorption Spectroscopy Using an Ultrashort Laser-Wakefield Accelerator Source, Phys. Rev. Lett. 123, 254801 (2019).

[31] T. Tajima and J. M. Dawson, Laser Electron-Accelerator, Phys. Rev. Lett. 43, 267 (1979).

[32] P. Chen and J. M. Dawson, The Plasma Wake Field Accelerator, AIP Conf. Proc. 130, 201 (1985).

[33] B. Hidding, A. Beaton, L. Boulton, S. Corde, A. Doepp, A. F. Habib, T. Heinemann, A. Irman, S. Karsch, G. Kirwan, A. Knetsch, G. G. Manahan, A. M. de la Ossa, A. Nutter, P. Scherkl, U. Schramm, and D. Ullmann, Fundamentals and Applications of Hybrid LWFA-PWFA, Appl. Sci. 9, 2626 (2019).

[34] S. Wilks, T. Katsouleas, J. M. Dawson, P. Chen, and J. J. Su, Beam Loading in Plasma-Waves, IEEE Trans. Plasma Sci. 15, 210 (1987).

[35] A. R. Maier, A. Meseck, S. Reiche, C. B. Schroeder, T. Seggebrock, and F. Grüner, Demonstration Scheme for a Laser-Plasma-Driven Free-Electron Laser, Phys. Rev. X 2 , 031019 (2012).

[36] M. E. Couprie, Panorama of New Generation of Accelerator Based Short Wavelength Coherent Light Sources, Nucl. Instrum. Methods Phys. Res., Sect. B 364, 4 (2015).

[37] G. G. Manahan, A. F. Habib, P. Scherkl, P. Delinikolas, A. Beaton, A. Knetsch, O. Karger, G. Wittig, T. Heinemann, Z. M. Sheng, J. R. Cary, D. L. Bruhwiler, J. B. Rosenzweig, and B. Hidding, Single-Stage Plasma-Based Correlated Energy Spread Compensation for Ultrahigh 6D Brightness Electron Beams, Nat. Commun. 8, 15705 (2017).

[38] B. Hidding, T. Königstein, J. Osterholz, S. Karsch, O. Willi, and G. Pretzler, Monoenergetic Energy Doubling in a Hybrid Laser-Plasma Wakefield Accelerator, Phys. Rev. Lett. 104, 195002 (2010).
[39] B. Hidding, G. Pretzler, J. B. Rosenzweig, T. Königstein, D. Schiller, and D. L. Bruhwiler, Ultracold Electron Bunch Generation via Plasma Photocathode Emission and Acceleration in a Beam-Driven Plasma Blowout, Phys. Rev. Lett. 108, 035001 (2012).

[40] A. M. de la Ossa, J. Grebenyuk, T. Mehrling, L. Schaper, and J. Osterhoff, High-Quality Electron Beams from BeamDriven Plasma Accelerators by Wakefield-Induced Ionization Injection, Phys. Rev. Lett. 111, 245003 (2013).

[41] A. M. de la Ossa, T. J. Mehrling, L. Schaper, M. J. V. Streeter, and J. Osterhoff, Wakefield-Induced Ionization Injection in Beam-Driven Plasma Accelerators, Phys. Plasmas 22, 093107 (2015).

[42] E. Esarey, C. B. Schroeder, and W. P. Leemans, Physics of Laser-Driven Plasma-Based Electron Accelerators, Rev. Mod. Phys. 81, 1229 (2009).

[43] V. Malka, Laser Plasma Accelerators, Phys. Plasmas 19, 055501 (2012).

[44] S. M. Hooker, Developments in Laser-Driven Plasma Accelerators, Nat. Photonics 7, 775 (2013).

[45] M. C. Downer, R. Zgadzaj, A. Debus, U. Schramm, and M. C. Kaluza, Diagnostics for Plasma-Based Electron Accelerators, Rev. Mod. Phys. 90, 035002 (2018).

[46] E. Esarey, P. Sprangle, J. Krall, and A. Ting, Overview of Plasma-Based Accelerator Concepts, IEEE Trans. Plasma Sci. 24, 252 (1996).

[47] W. Lu, C. Huang, M. Zhou, W. B. Mori, and T. Katsouleas, Nonlinear Theory for Relativistic Plasma Wakefields in the Blowout Regime, Phys. Rev. Lett. 96, 165002 (2006).

[48] A. Pukhov and J. Meyer-ter-Vehn, Laser Wake Field Acceleration: The Highly Non-linear Broken-Wave Regime, Appl. Phys. B 74, 355 (2002).

[49] I. Kostyukov, A. Pukhov, and S. Kiselev, Phenomenological Theory of Laser-Plasma Interaction in "Bubble" Regime, Phys. Plasmas 11, 5256 (2004).

[50] See Sec. I in Supplemental Material [51].

[51] See Supplemental Material at http://link.aps.org/ supplemental/10.1103/PhysRevX.10.041015 for additional information on beam loading physics, an explanation of the central importance of the beam current, discussions on the experimental setup, and further details on the data analysis.

[52] H. Ding, A. Döpp, M. Gilljohann, J. Götzfried, S. Schindler, L. Wildgruber, G. Cheung, S. M. Hooker, and S. Karsch, Nonlinear Plasma Wavelength Scalings in a Laser Wakefield Accelerator, Phys. Rev. E 101, 023209 (2020).

[53] Details on the simulations underlying Fig. 1 are given in Supplemental Material [51].

[54] W. Lu, C. Huang, M. Zhou, M. Tzoufras, F. S. Tsung, W. B. Mori, and T. Katsouleas, A Nonlinear Theory for Multidimensional Relativistic Plasma Wave Wakefields, Phys. Plasmas 13, 056709 (2006).

[55] C. Rechatin, X. Davoine, A. Lifschitz, A. B. Ismail, J. Lim, E. Lefebvre, J. Faure, and V. Malka, Observation of Beam Loading in a Laser-Plasma Accelerator, Phys. Rev. Lett. 103, 194804 (2009).

[56] C. Rechatin, J. Faure, X. Davoine, O. Lundh, J. Lim, A. Ben-Ismail, F. Burgy, A. Tafzi, A. Lifschitz, E. Lefebvre, and V. Malka, Characterization of the Beam Loading Effects in a Laser Plasma Accelerator, New J. Phys. 12, 045023 (2010). 
[57] E. Guillaume, A. Döpp, C. Thaury, A. Lifschitz, J. P. Goddet, A. Tafzi, F. Sylla, G. Iaquanello, T. Lefrou, P. Rousseau, K. T. Phuoc, and V. Malka, Physics of FullyLoaded Laser-Plasma Accelerators, Phys. Rev. Accel. Beams 18, 061301 (2015).

[58] J. P. Couperus, R. Pausch, A. Köhler, O. Zarini, J. M. Krämer, M. Garten, A. Huebl, R. Gebhardt, U. Helbig, S. Bock, K. Zeil, A. Debus, M. Bussmann, U. Schramm, and A. Irman, Demonstration of a Beam Loaded Nanocoulomb-Class Laser Wakefield Accelerator, Nat. Commun. 8, 487 (2017).

[59] M. Tzoufras, W. Lu, F. S. Tsung, C. Huang, W. B. Mori, T. Katsouleas, J. Vieira, R. A. Fonseca, and L. O. Silva, Beam Loading by Electrons in Nonlinear Plasma Wakes, Phys. Plasmas 16, 056705 (2009).

[60] K. H. Pae, I. W. Choi, and J. Lee, Self-Mode-Transition from Laser Wakefield Accelerator to Plasma Wakefield Accelerator of Laser-Driven Plasma-Based Electron Acceleration, Phys. Plasmas 17, 123104 (2010).

[61] S. Corde, C. Thaury, K. T. Phuoc, A. Lifschitz, G. Lambert, J. Faure, O. Lundh, E. Benveniste, A. Ben-Ismail, L. Arantchuk, A. Marciniak, A. Stordeur, P. Brijesh, A. Rousse, A. Specka, and V. Malka, Mapping the X-Ray Emission Region in a Laser-Plasma Accelerator, Phys. Rev. Lett. 107, 215004 (2011).

[62] P. E. Masson-Laborde, M. Z. Mo, A. Ali, S. Fourmaux, P. Lassonde, J. C. Kieffer, W. Rozmus, D. Teychenne, and R. Fedosejevs, Giga-electronvolt Electrons due to a Transition from Laser Wakefield Acceleration to Plasma Wakefield Acceleration, Phys. Plasmas 21, 123113 (2014).

[63] M. Heigoldt, A. Popp, K. Khrennikov, J. Wenz, S. W. Chou, S. Karsch, S. I. Bajlekov, S. M. Hooker, and B. Schmidt, Temporal Evolution of Longitudinal Bunch Profile in a Laser Wakefield Accelerator, Phys. Rev. Accel. Beams 18, 121302 (2015).

[64] Y. Wu, C. Yu, Z. Qin, W. Wang, Z. Zhang, R. Qi, K. Feng, L. Ke, Y. Chen, C. Wang, X. Yang, Y. Leng, J. Liu, R. Li, and $\mathrm{Z}$. Xu, Energy Enhancement and Energy Spread Compression of Electron Beams in a Hybrid Laser-Plasma Wakefield Accelerator, Appl. Sci. 9, 2561 (2019).

[65] H. Suk, N. Barov, J. B. Rosenzweig, and E. Esarey, Plasma Electron Trapping and Acceleration in a Plasma Wake Field Using a Density Transition, Phys. Rev. Lett. 86, 1011 (2001).

[66] H. Suk, H. J. Lee, and I. S. Ko, Generation of High-Energy Electrons by a Femtosecond Terawatt Laser Propagating through a Sharp Downward Density Transition, J. Opt. Soc. Am. B 21, 1391 (2004).

[67] K. Schmid, A. Buck, C. M. S. Sears, J. M. Mikhailova, R. Tautz, D. Herrmann, M. Geissler, F. Krausz, and L. Veisz, Density-Transition Based Electron Injector for Laser Driven Wakefield Accelerators, Phys. Rev. Accel. Beams 13, 091301 (2010).

[68] E. Guillaume, Control of Electron Injection and Acceleration in Laser-Wakefield Accelerators, Ph.D. thesis, Ecole Polytechnique, 2015.

[69] H.-E. Tsai, K. K. Swanson, S. K. Barber, R. Lehe, H.-S. Mao, D. E. Mittelberger, S. Steinke, K. Nakamura, J. van Tilborg, C. Schroeder, E. Esarey, C. G. R. Geddes, and W. Leemans, Control of Quasi-monoenergetic Electron Beams from Laser-Plasma Accelerators with Adjustable Shock Density Profile, Phys. Plasmas 25, 043107 (2018).

[70] K. Schmid and L. Veisz, Supersonic Gas Jets for LaserPlasma Experiments, Rev. Sci. Instrum. 83, 053304 (2012).

[71] T. Kurz, J. P. Couperus, J. M. Krämer, H. Ding, S. Kuschel, A. Köhler, O. Zarini, D. Hollatz, D. Schinkel, R. D’Arcy, J.-P. Schwinkendorf, J. Osterhoff, A. Irman, U. Schramm, and S. Karsch, Calibration and Cross-Laboratory Implementation of Scintillating Screens for Electron Bunch Charge Determination, Rev. Sci. Instrum. 89, 093303 (2018).

[72] A. Döpp, C. Thaury, E. Guillaume, F. Massimo, A. Lifschitz, I. Andriyash, J. P. Goddet, A. Tazfi, K. T. Phuoc, and V. Malka, Energy-Chirp Compensation in a Laser Wakefield Accelerator, Phys. Rev. Lett. 121, 074802 (2018).

[73] R. Lehe, M. Kirchen, I. A. Andriyash, B. B. Godfrey, and J.-L. Vay, A Spectral, Quasi-cylindrical and DispersionFree Particle-in-Cell Algorithm, Comput. Phys. Commun. 203, 66 (2016).

[74] W. Lu, M. Tzoufras, C. Joshi, F. Tsung, W. Mori, J. Vieira, R. Fonseca, and L. Silva, Generating Multi-GeV Electron Bunches Using Single Stage Laser Wakefield Acceleration in a 3D Nonlinear Regime, Phys. Rev. Accel. Beams 10, 061301 (2007).

[75] A. Pukhov, S. Gordienko, S. Kiselev, and I. Kostyukov, The Bubble Regime of Laser-Plasma Acceleration: Monoenergetic Electrons and the Scalability, Plasma Phys. Controlled Fusion 46, B179 (2004).

[76] M. F. Gilljohann, H. Ding, A. Döpp, J. Götzfried, S. Schindler, G. Schilling, S. Corde, A. Debus, T. Heinemann, B. Hidding, S. M. Hooker, A. Irman, O. Kononenko, T. Kurz, A. M. de la Ossa, U. Schramm, and S. Karsch, Direct Observation of Plasma Waves and Dynamics Induced by Laser-Accelerated Electron Beams, Phys. Rev. X 9, 011046 (2019).

[77] There are different ways to define scaling for laser wakefields. Pukhov et al. [75], for instance, introduce a similarity parameter $\alpha=n_{e} /\left(a_{0} n_{\mathrm{cr}}\right)$, while our approach uses direct scaling of all length scales in the plasma similar to Lu et al. [74].

[78] K. Schmid, L. Veisz, F. Tavella, S. Benavides, R. Tautz, D. Herrmann, A. Buck, B. Hidding, A. Marcinkevicius, U. Schramm, M. Geissler, J. Meyer-ter-Vehn, D. Habs, and F. Krausz, Few-Cycle Laser-Driven Electron Acceleration, Phys. Rev. Lett. 102, 124801 (2009).

[79] D. Guénot, D. Gustas, A. Vernier, B. Beaurepaire, F. Böhle, M. Bocoum, M. Lozano, A. Jullien, R. Lopez-Martens, A. Lifschitz, and J. Faure, Relativistic Electron Beams Driven by kHz Single-Cycle Light Pulses, Nat. Photonics 11, 293 (2017).

[80] T. André et al., Control of Laser Plasma Accelerated Electrons for Light Sources, Nat. Commun. 9, 1334 (2018).

[81] A. Buck, J. Wenz, J. Xu, K. Khrennikov, K. Schmid, M. Heigoldt, J. M. Mikhailova, M. Geissler, B. Shen, F. Krausz, S. Karsch, and L. Veisz, Shock-Front Injector for HighQuality Laser-Plasma Acceleration, Phys. Rev. Lett. 110, 185006 (2013).

[82] J. Faure, C. Rechatin, A. Norlin, A. Lifschitz, Y. Glinec, and V. Malka, Controlled Injection and Acceleration of Electrons in Plasma Wakefields by Colliding Laser Pulses, Nature (London) 444, 737 (2006). 
[83] J. Wenz, A. Döpp, K. Khrennikov, S. Schindler, M. F. Gilljohann, H. Ding, J. Götzfried, A. Buck, J. Xu, M. Heigoldt, W. Helml, L. Veisz, and S. Karsch, Dual-Energy Electron Beams from a Compact Laser-Driven Accelerator, Nat. Photonics 13, 263 (2019).

[84] T. Kurz et al., Demonstration of a Compact Plasma Accelerator Powered by Laser-Accelerated Electron Beams, arXiv:1909.06676.

[85] This approach assumes that the beam at the exit has a divergence of at least the vacuum focus $(f / 25)$. In reality, this estimate is lower because of self-focusing, which induces additional wavefront curvature. Note that this estimation assumes that the beams are far away from the focus, which is not necessarily the case for the electron beam in our conditions. Close to focus, the laser intensity of a Gaussian beam evolves as $I(z) \propto w(z)^{-2}$ with $w(z)=$ $w_{0} \sqrt{1+\left(z / z_{R}\right)^{2}}, \quad z_{R}=\pi w_{0}^{2} / \lambda$ being the Rayleigh length and $w_{0}$ denotes the focal spot radius. The electron bunch evolves according to $\sigma_{r}=\sigma_{r 0} \sqrt{1+(z / \tilde{\beta})^{2}}$ with $\tilde{\beta}=\sigma_{r 0}^{2} \gamma / \epsilon_{n}, \quad \sigma_{r 0}$ being the focal spot radius of the electron bunch and $\epsilon_{n}$ denoting the normalized emittance.

[86] S. Chou, J. Xu, K. Khrennikov, D. E. Cardenas, J. Wenz, M. Heigoldt, L. Hofmann, L. Veisz, and S. Karsch, Collective
Deceleration of Laser-Driven Electron Bunches, Phys. Rev. Lett. 117, 144801 (2016).

[87] M. Kirchen, R. Lehe, B. B. Godfrey, I. Dornmair, S. Jalas, K. Peters, J. L. Vay, and A. R. Maier, Stable Discrete Representation of Relativistically Drifting Plasmas, Phys. Plasmas 23, 100704 (2016).

[88] R. Lehe, M. Kirchen, B. B. Godfrey, A. R. Maier, and J.-L. Vay, Elimination of Numerical Cherenkov Instability in Flowing-Plasma Particle-in-Cell Simulations by Using Galilean Coordinates, Phys. Rev. E 94, 053305 (2016).

[89] C. Thaury, E. Guillaume, A. Döpp, R. Lehe, A. Lifschitz, K. T. Phuoc, J. Gautier, J. P. Goddet, A. Tafzi, A. Flacco, F. Tissandier, S. Sebban, A. Rousse, and V. Malka, Demonstration of Relativistic Electron Beam Focusing by a LaserPlasma Lens, Nat. Commun. 6, 6860 (2015).

[90] R. Lehe, C. Thaury, A. Lifschitz, J. M. Rax, and V. Malka, Transverse Dynamics of an Intense Electron Bunch Traveling through a Pre-ionized Plasma, Phys. Plasmas 21, 043104 (2014).

[91] More details on the setup are given in Supplemental Material [51].

[92] A. Loulergue, M. Labat, C. Evain, C. Benabderrahmane, V. Malka, and M. E. Couprie, Beam Manipulation for Compact Laser Wakefield Accelerator Based Free-Electron Lasers, New J. Phys. 17, 023028 (2015). 